\title{
Modeling and Case Study of the Production Line Layout System
}

\author{
Yong Zhao, Xiaoyong Pan, Chun Chuan Li, Guohong Yao, Jiang Wu, Xin Fu*, Dong Lai, Dong Li \\ and Quanwei Zhang
}

\author{
Changhong Electric Co.,Ltd. Mianyang, SiChuan 621000, China
}

\begin{abstract}
This paper deals with the production line layout problem. A study on this problem indicates there is a lack of research on the production line design issue. Traditional methodology on this problem mainly depends on the designer's experience and calls for theory analysis and quantitative study. To control the cost and optimize the resource utilization of enterprises, this paper develops a multi-stage integer programming model to describe the production line layout problem, and proposes a heuristic algorithm to solve the problem. A practical example illustrates the effectiveness of the model.
\end{abstract}

Keywords: Flow line, Production layout, Integer programming, Ant-colony algorithm.

\section{INTRODUCTION}

The production line layout problem is one of the most important research areas in industry. This problem concerns with the production process, the utility of facilities, the life of equipment and the production safety issues. Nowadays, the market competence has become even fierce, and in order to keep up with the competence, many enterprises introduce concepts such as JIT, lean production, etc., to meet the various personalized demand of customers. In these topics, the research of optimizing production process is one of the most important issues.

Most research on optimizing production process has been conducted on the quantity analysis, such as [1-4]. However, they study mainly on problems such as job scheduling and production strategies on a given production line layout. There is little literature on how to generate the layout, which related to the sequence of work stations, the geometry structure of production lines, the deployment of resources, etc. The profits under different layouts can vary dramatically. The heart of this problem is to design algorithms for the layout issues through analysis of production categories, batches, and processes to maximize the return of investments. Spinellis and Papadopoulos [5] proposes a simulated annealing algorithm to solve the allocation problem of work stations and buffers in a serial line; [6] Benjaafar et al. studies the machine layout problem under dynamic and uncertain circumstances [7]. Kuroda and Tomita studies the machine layout problem on a cellular flow line under stochastic machine break down situations. Papadopoulos et al. [8] studies the layout of buffers and work stations. They focus mainly on the layout of machines, work stations, and buffers, and with little consideration of re-arrangement of production lines.

*Address correspondence to this author at the Changhong Electric Co.,Ltd. Mianyang, SiChuan 621000, China; Tel: +86-0816-2418609;

E-mail: xin.fu@changhong.com
On the other hand, when the factory produces varied products, we have to take consideration of production scheduling problem. Many research studies have been carried out on this topic, such as Johnson [9], Quadt and Kuhn [10], Ribas et al. [11], Urlings et al. [12], Haouari and Hidri [13], Hidri and Haouari et al. [14, 15].

This paper studies the production line layout problem with diversified products. The objective is to determine the layout of production lines subjected to the constraints such as capital, workers, and space.

\section{PRODUCTION LINE LAYOUT SYSTEM MODELING}

Production line layout system has many specific geometric structures, but the basics are the serial and parallel lines.

Most traditional lines are serial lines. In this layout, the work is split into many sequential tasks, and if anyone fails to complete his task in time, the work behind him will all stop; if the tasks are not balanced among workers, there will be bottlenecks and cause loss; most importantly, the serial line is vulnerable to fluctuations, which is even worse when there are few of buffers, as in Fig. (1).

The parallel line consists of many independent parallel production lines, which can produce the same or different products at the same time, as in Fig. (2). However, each production line should be equipped with the equipment and tools, which is a huge investment and require large maintenance cost; the intensity and complexity of material transportation in the parallel lines lead to a high logistic cost; the demand for warehouses are also stricter.

In order to combine the advantage of low cost and easy implementation of serial production line, as well as the ability to handle fluctuations of production in parallel production line, we propose a general production line structure to meet the demand of variable specialized customer needs and the changeable markets. 


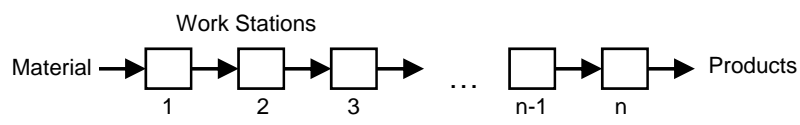

Fig. (1). The serial line.

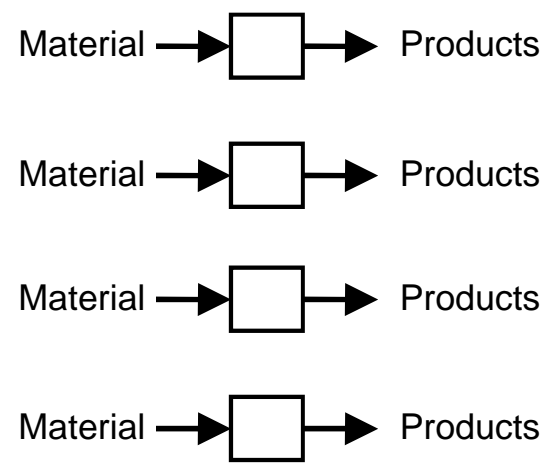

Fig. (2). The parallel line.

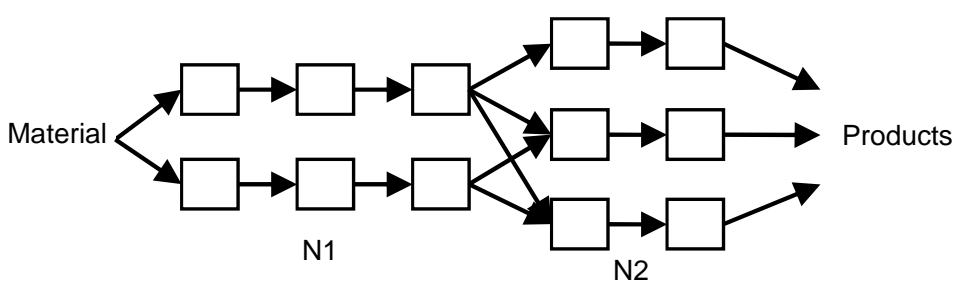

Fig. (3). The hybrid line.

\subsection{Layout Modeling}

Assume that the production process contains $L_{L}$ stages, the total number of workers is $E$. Use $\{N, S, A\}$ to describe the system. $X=\{N, S\}$ is the layout of production lines, where $n_{l} \in N$ is the number of parallel lines, and $s_{l} \in S$ is the number of workers in each line at the $l$ th stage. $A$ is the schedule matrix, and $a_{k, l, i} \in A$ is the quantity of $k$ th product scheduled to the $i$ th line at the $l$ th stage.

When $\mathrm{N}$ is smaller, $\mathrm{S}$ is bigger, the production line layout is similar to the serial line, which is typical in flow-line manufactories; when $\mathrm{S}$ is smaller and $\mathrm{N}$ is larger, the production line is similar to cell line, which is typical in many modern lean production manufactories. The serial line and the cell line are both specific structure of the general structure, which we call the hybrid line, as Fig. (3).

To decrease the cost and increase the flexibility of the production line, we require detailed analysis. Take the first stage of the production process for example. The number of production lines influences the time loss rate of batch changes, the number of material transfer lines, the number of tools and equipment; the number of work stations influences the work load, the tack time, the production rate, the balance rate, etc. They also affect the space needed, the energy assumption, and the number of work-in-process.

As we know, when the layout is in the form of serial line, the production cost is relatively low, but the time loss caused by frequent change which comes from the variety of market demand is high, and the production process tends to be unreliable; when the layout is in the form of parallel line, the production loss caused by fluctuation is relatively low, but the equipment investment, the energy consumption and the logistic cost is high. To balance the advantages and disadvantages, we build a model to describe the system.

\subsection{Objective Function}

To maximize Input-Output Ratio, the overall objective function of the optimization model is:

$\max \frac{M(N, S, A)}{C(N, S, A)}$

$C\{N, S, A\}$ represents the function of the total investment of the production, which is related to the production layout $\{N, S, A\}$. It consists of raw material cost, total labor cost, logistics cost, land cost, equipment cost, energy consumption and occupancy cost. $M\{N, S, A\}$ represents the total production sales revenue, which is related to the production capacity of designated production layout, which means $\{N, S, A\}$ :

$M_{T}=\sum_{k=1}^{K} Q_{k} M_{k}$

\subsection{Cost Analysis}

Cost consists of 7 parts:

$C(N, S, A)=\sum_{i=1}^{7} C_{i}(N, S, A)$ 


\section{(1) Raw Material Cost}

Raw material cost is the purchasing price of total raw material based on BOM :

$$
C_{1}(N, S, A)=\sum_{k=1}^{K} C_{1, k} Q_{k}
$$

$C_{1, k}$ is Raw material cost of the $k$ th production. $Q_{k}$ stands production quantity of the $k$ th production within P-Q info:

$Q_{k}=\sum_{n=1}^{N_{1}} a_{k, n}$

$N_{1}$ is the number of production line of the first stage.

\section{(2)Total Labor Cost}

Total labor cost is the sum of each and every worker's time rate wage, which is the product of hourly wage and working time (hours):

$C_{2}(N, S, A)=M_{0} \sum_{k=1}^{K} \sum_{l=1}^{L} T_{H, k, l} Q_{k}+M_{0} N_{G} T_{\text {Change }}$

$T_{H, k, l}$ is the actual working hours of the $k$ th product in the lth stage:

$$
\begin{gathered}
T_{H, k, l}=T_{e f f, k, l}+\frac{T_{e f f, k, l}}{t_{k, l}}\left(\Delta t_{k, l}+t_{\text {stop }, k, l}\right) \\
T_{\text {eff } k, l}
\end{gathered}
$$

$T_{e f f, k, l}$ is the eff,k,l standard working hours of the $k$ th product in the $l$ th stage. $\quad t_{k, l}$ is the number of workers of each production line of the $k$ th product in the $l$ th stage. $M_{0}$ stands the hourly wage. $t_{s t o p, k, l}$ is the expected blockage time caused by each workstation's misoperation. $\Delta t_{k, l}$ is the transport time between adjacent workstations of the $k$ th production in the lth stage. $M_{0} N_{G} T_{\text {Change }}$ stands the loss of labor cost when the production process pauses.

\section{(3) Logistics Costs}

There are two kinds of path of the logistics path: common path (such as shop entrance to the staging area) and proprietary path (such as the staging area to the production line).

Logistics cost is the product of workers' hourly wage and total labor. Total labor is the result of the total logistics equivalent divided by the single worker's average transportation capability.

$C_{3}(N, S, A)=M_{0} \frac{W_{L}}{W_{H}}$

$W_{L}$ is the total logistics equivalent. $W_{H}$ is one worker's average logistics equivalent per unit time,

$W_{H}=W L_{0}+\sum_{j=1}^{N_{1}} W_{j} D_{j}$

$L_{0}$ is the distance of common path, for example, the distance between warehouse and the raw materials dump in workshop.

$W=\sum_{j=1}^{N_{1}} W_{j}$
$W$ represents the total raw materials weight prepared for the first stage.

$W_{j}=\sum_{k=1}^{K} a_{j, k} D_{j}$

$W_{j}$ represents the total raw materials weight prepared for the $j$ th production line in the first stage.

$D_{j}=(j-1) L_{1}+\frac{S_{1} L_{H}}{2}$

$D j$ represents the average transport distance between the raw materials dump in workshop and each production line of the first stage. $L_{l}$ represents the distance between adjacent production lines in the $l$ th stage. $L_{H}$ represents the distance between adjacent workstations, as shown in Fig. (4).

\section{(4)Land Cost}

$C_{4}(N, S, A)=\left(\beta_{1,1} \% c_{1,1}+\beta_{1,2} \% c_{1,2}\right) S_{T}$

$c_{1,1}$ is acquisition costs per unit land square. $c_{1,2}$ is plant construction costs per unit land square. $\beta_{1,1} \%$ is the depreciation coefficient of the land through the producing time of a batch of products. $\beta_{1,2} \%$ is the depreciation coefficient of plant through the producing time of a batch of products. $S_{T}$ is the plant square.

$S_{T}=(1+\alpha \%) \sum_{l=1}^{4} N_{l} L_{l} S_{l} L_{H}$

$\alpha \%$ represents the percentage of non-production square of the total plant square.

\section{(5) Equipment Cost}

The equipment cost consists of production equipment cost (such as production lines, motors, etc.) and auxiliary equipment cost (such as logistics transport vehicles, pallets, etc.), and the ongoing maintenance costs:

$C_{5}(N, S, A)=\left(\beta_{2} \% c_{2} L_{T}+\beta_{3} \% c_{3} L_{T}\right)(1+\varepsilon)$

$c_{2}$ is the average production line cost per unit length. $c_{3}$ is the average auxiliary equipment cost of production line per unit length. $\beta_{2} \%$ is the depreciation coefficient of production equipment through the producing time of a batch of products. $\beta_{3} \%$ is the depreciation coefficient of auxiliary equipment through the producing time of a batch of products. $\varepsilon$ is the percentage of equipment maintenance costs to total equipment costs.

$L_{T}=\sum_{l=1}^{4} N_{l} S_{l} L_{H}$

$L_{T}$ represents the length of total production line, without considering the transmission belt.

\section{(6) Energy Consumption Cost}

Energy consumption consists of two parts: the plant lighting and production line power. Energy consumption cost is the product of energy consumption power and running time. 


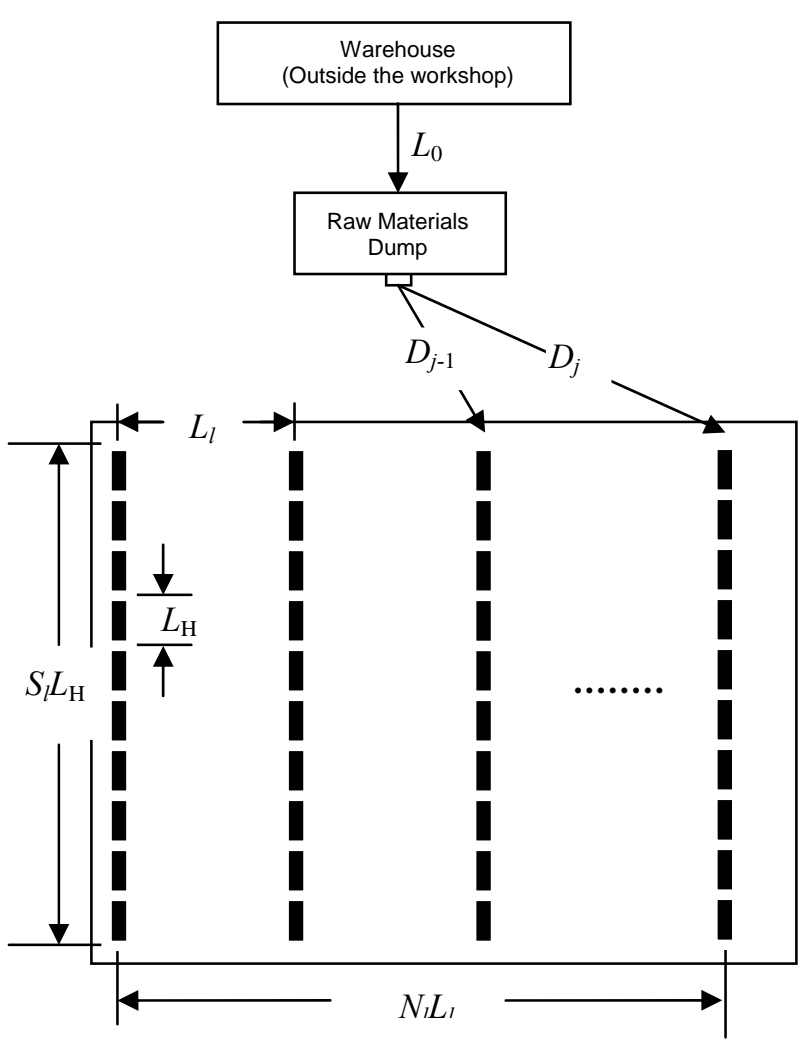

Fig. (4). Logistic.

$$
C_{6}(N, S, A)=T_{\text {total }}\left(\rho S_{T}+\eta L_{T}\right)
$$

$\rho$ is the cost of energy consumption of plant lighting per unit area and per unit time. $\eta$ represents the cost of energy consumption of production line operating per unit time.

$T_{\text {total }}=T_{\text {total }, 1}+T_{\text {Change }}$

$T_{\text {total }}$ represents total producing time to complete an entire batch of products.

$T_{\text {total }, 1}=T_{1-4}+T_{5}$

$T_{\text {total, } 1}$ represents effective producing time, where:

$T_{1-4}=\sum_{k=1}^{K} \frac{Q_{k}}{Q}\left(T_{k, 1}+T_{k, 2}+T_{k, 3}+T_{k, 4}\right)$

$T_{1-4}$ represents average production cycle time to complete one product.

$T_{k, l}=S_{l}\left(t_{k, l}+\Delta t_{k, l}+t_{s t o p, k, l}\right)$

$T_{5}=\frac{Q \sum_{k=1}^{K} Q_{k}\left(t_{k, 1}+\Delta t_{k, 1}+t_{s t o p, k, l}\right)}{N_{1}}$

$T_{5}$ represents production time to complete all products apart from the first product.
$T_{\text {Change }}=\frac{1}{N_{1}} N_{C} T_{C}$

$T_{\text {change }}$ represents total exchange time during producing. $T_{c}$ is total working time influenced by exchange. $N_{c}$ is exchange frequency:

$$
N_{C}=\sum_{j=1}^{N_{1}} \sum_{k=1}^{K} I_{j, k}, \quad I_{j, k}= \begin{cases}1, & a_{j, k}>0 \\ 0, & a_{j, k}=0\end{cases}
$$

\section{(7) Occupancy Cost}

Occupancy cost is the cost of capital occupied by all semi-finished and finished products.

$C_{7}=\left(C_{M} t_{\text {before }}+C_{\text {WIP }} t_{\mathrm{WIP}}+C_{\text {Store }} t_{\text {after }}\right) \zeta \%$

$C_{M}$ is raw material cost.

$C_{W I P}=\left(T_{1-4} \frac{N_{1}}{t_{k, 1}+\Delta t_{k, 1}+t_{s t o p, k, 1}}\right)\left(\sum_{k=1}^{K} \frac{Q_{k}}{Q} P_{k}\right)$

$C_{W I P}$ is cost of WIP. $C_{\text {stroe }}$ is cost of storage. $t_{\text {before }}$ is the raw material preparing time before producing. $t_{\text {wip }}$ is the producing time. $t_{\text {after }}$ is the storage time after producing completed. $\zeta \%$ is interest rate.

\subsection{Constraint Analysis}

In our model, the number of workers is always the same in different production lines in a designated stage, even if producing different kinds of products:

$\frac{T_{e f f, k_{1}, l}}{T_{e f f, k_{2}, l}}=\frac{t_{k_{1}, l}}{t_{k_{2}, l}}$

$T_{\text {eff }, k, l}$ stands the time for the $k$ th product in the $l$ th stage. $t_{k, l}$ is the cycle time for the $k$ th product in the $l$ th stage.

In order to ensure a smooth flow of products, the quantity of products completed per unit time in different stage must be the same and satisfied the pace constraint:

$$
\begin{aligned}
& \frac{N_{1}}{t_{k, 1}+\Delta t_{k, 1}+t_{s t o p, k, 1}}=\frac{N_{2}}{t_{k, 2}+\Delta t_{k, 2}+t_{s t o p, k, 2}} \\
& =\frac{N_{3}}{t_{k, 3}+\Delta t_{k, 3}+t_{s t o p, k, 3}}, k=1,2, \cdots, K \\
& \frac{N_{1}}{\sum_{k=1}^{K} \frac{Q_{k}}{Q}\left(t_{k, 1}+\Delta t_{k, 1}+t_{\text {stop }, k, 1}\right)} \leq \frac{Q}{T_{P Q}}, \quad k=1,2, \cdots, K
\end{aligned}
$$

The quantity of all kinds of products completed in average aging time in the first stage is equal to the number of aging district location.

$N_{2} S_{2}=\left(\sum_{k=1}^{K} \frac{Q_{k}}{Q} T_{k, 1}\right)\left(\sum_{k=1}^{K} \frac{Q_{k}}{Q} \frac{N_{1}}{t_{k, 1}+\Delta t_{k, 1}+t_{s t o p, k, l}}\right)$ 
In addition, consider the order constraint on producing time and product quantity:

$$
\begin{aligned}
& T_{\text {total }}=T_{\text {total }, 1}+T_{\text {chang }}<T_{P Q} \\
& \sum_{j=1}^{N 1} Q_{j, k}=Q_{k} \\
& \sum_{K=1}^{K} Q_{k}=Q
\end{aligned}
$$

$T_{P Q}$ is the total producing time within the $\mathrm{P}-\mathrm{Q}$ info.

\subsection{Algorithm}

The model is a nonlinear and integer programming model. We use a heuristic method and the ant-colony algorithm to solve the problem.

We can produce feasible regions for $N$ and $S$ with the model constrains. The procedure to solve the problem is:

(1) Initialize $X=X^{(0)}$. Define the iteration number $i=0$.

(2) Use the ant-colony algorithm to produce the schedule matrix $A$ corresponding to $X^{(i)}$.

(3) Use the heuristic algorithm to calculate $X^{(i+1)}$.

(4) If $i \leqslant N^{T}$, transfer to step (2); else terminate the calculation.

The procedure to get $X$ is:

(1) Define the iteration number $i=0$.

(2) Define the stage number $l=1$.

(3) Find a better $\left\{n_{l}, s_{l}\right\}$ value, defined as $\left\{n_{l}, S_{l}\right\}^{(l)}$ (in order to avoid local optimal, we let the value be a random one with probability $\rho$ ), and update the schedule matrix .

(4) If $l \leq L$, then let $l=l+1$, and transfer to step (3); else let $i=i+1$.

(5) If $i \leq N^{H}$, then transfer to (2); else terminate the calculation.

Give $N_{l}$ production lines, $K$ types of products and the number of each products, We employ any-colony algorithm to generate the schedule matrix:

$$
A=\left(a_{i j}\right)_{k, N l}
$$

where $a_{k, j}$ represents the work load of the $k$ th products assigned to the $j$ th production line. The total line change time is minimized and the workloads among different production lines are balanced. The procedure to calculate the schedule matrix $A$ for $X$ is:

(1)Initialize algorithm parameters.

(2)Initialize $W$. Choose $n_{l}$ products with longest production time as the 1st task. Use a tabu list to build the following routes, and construct a complete schedule matrix.

(3)Update the pheromone. Calculate the total production time of each line, and get the best iteration solutions and the current best solution. Update the pheromone according to the information.
(4)If the current best solution does not improve for consequent $n$ iterations, we shall initialize the pheromone to 0 and transfer to step (3) in order to jump out of the local optimal.

(5)If the total iteration time reaches $N^{A}$, then terminate the calculation.

\section{THE EVALUATION METHOD BASED ON RSR}

In the workshop, five indicator indexes are our primary concerns: the investment of production line $\left(I_{P L I}\right)$, the output per unit time per unit area $\left(I_{U A P H}\right)$, the output per unit time per unit person $\left(I_{U P P H}\right)$, the production fluctuation rate $\left(I_{F R}\right)$, and the quantity of WIP $\left(I_{W I P}\right)$.

\section{(1) Design of original data table}

Make five assessment indexes of each evaluation object into the original data table.

(2) Rank: Rank each index. The high-good indexes rank from small to large, while the low-good indexes rank from large to small, and the indexes of the same indicator have the same value share the mean rank. The high-good index means the value the bigger the better, contrary to the low-good index. Therefore, $I_{U A P H} 、 I_{U P P H}$ belongs to high-good index, the larger the index value, the higher the rank; $I_{P L I}, I_{F R}, I_{W I P}$ belongs to the low-good index, the larger the index value, the lower the rank.

(3) Calculate the rank sum ratio: in a matrix of $n$ (number of objects) *m column (number of indexes), the calculating formula of RSR:

$R S R_{i}=\frac{1}{m \cdot n} \sum_{j=1}^{m} R_{i j} i=1,2, \cdots, n ; j=1,2,3, \cdots m$

$R_{i j}$ is the rank of the element of the $i$ th row and the $j$ th column. Sort the evaluation objects according to the RSR value.

(4) To the different weights of each assessment index, calculate Weighted Rank Sum Ratio, the calculation formula as follows:

$W R S R_{i}=\frac{1}{n} \sum_{j=1}^{m} \omega_{j} R_{i j} i=1,2, \cdots, n ; j=1,2,3, \cdots m$

$R_{i j}$ is the rank of the element of the $i$ th row and the $j$ th column. $\omega_{j}$ is the weight of the $j$ th evaluation index, satisfied $\sum_{j=1}^{m} \omega_{j}=1$

Five indicators do not affect the productivity in the same way; hence the weight of each index has to be determined first. The calculation method of weight is based on AHP as follows:

Begin with the second layer of the hierarchy model,

Each factor which will influence the upper layer of the same layer should compare with each other, end with the last layer. Compare that to the importance level of the criterion, build "pair comparison matrix" quantitatively according to the scale of grade (i.e.: "importance" is treated as a unified format, the meaning of each level shown in Table 1) 
Table 1. Scale of Grade

\begin{tabular}{|c|c|}
\hline Scale & Description \\
\hline \hline 1 & The two factors has the same importance \\
\hline 3 & The former is somewhat more important than the latter \\
\hline 5 & The former is obviously more important than the latter \\
\hline 7 & The former is strong more important than the latter \\
\hline 9 & The former is extremely more important than the latter \\
\hline 4,8 & The middle value between the adjacent scales \\
\hline reciprocal & $\begin{array}{c}\text { If the importance of factor } \mathrm{i} \text { to factor } \mathrm{j} \text { ratio is } \mathrm{a}_{\mathrm{ij}}, \mathrm{The} \\
\text { importance of factor } \mathrm{j} \text { and factor } \mathrm{i} \text { ratio is } \mathrm{a}_{\mathrm{ij}}=1 / \mathrm{a}_{\mathrm{ji}}\end{array}$ \\
\hline
\end{tabular}

Compare the influence degree of five factors include $I_{U A P H}, I_{U P P H} I_{F W}, I_{W I P}, I_{P L I}$ to the target layer factors respectively. The corresponding pairwise comparison matrix is:

$$
A=\left[\begin{array}{ccccc}
1 & 1 & 5 & 5 & \frac{1}{3} \\
1 & 1 & 5 & 7 & \frac{1}{5} \\
\frac{1}{5} & \frac{1}{5} & 1 & 3 & \frac{1}{9} \\
\frac{1}{5} & \frac{1}{7} & \frac{1}{3} & 1 & \frac{1}{9} \\
3 & 5 & 9 & 9 & 1
\end{array}\right]
$$

Calculate relative weight of each factor in pair comparison matrix to each criterion. Pairwise comparison matrix $A$ corresponding to the eigenvector $W$ of the maximum eigenvalue $\lambda$ max. After normalization, it is the sort weight of relative importance from the corresponding factors in the same level to the upper level. Calculate the eigenvector $W$ of maximum eigenvalue $\lambda_{\text {max }}$ by using MatLab method:

$[Y, D]=\operatorname{eig}(A)$

The maximum eigenvalue of eigenvector of $A$ is (-0.313 $0.3115-0.0910-0.0552-0.8909)^{\mathrm{T}}$. Through normalization here comes the weight vector of the factors of criterion layer:

$$
W=\left(\begin{array}{lllll}
0.1884 & 0.1874 & 0.0548 & 0.0332 & 0.5361
\end{array}\right)^{\mathrm{T}}
$$

In some cases, the pairwise comparison matrix constructed by comparison of two factors may get some inconsistent conclusions. It is difficult to achieve absolute consistency, and certain deviation is allowed with consistency check by calculating the consistency ratio $(C R)$ :

$C R=\frac{C I}{R I}$

$R I$ is the random consistency index, which value can be found in Table 2:

$C I=\left(\lambda_{\text {max }}-n\right) /(n-1)$

(41) $\lambda_{\max }$ is the largest eigenvalue of the pairwise comparison matrix, $n$ is the number of pairwise comparison factor, as the number of rows of the pairwise comparison matrix.

When $C R \leq 0.1$, the consistency of the pairwise comparison matrix is acceptable; When $C R>0.1$, the pairwise comparison matrix should make appropriate correction. Calculated in accordance with the criteria, the largest eigenvalue of the consistent index is $5.28, \mathrm{CI}=0.07$, And find the corresponding consistency average random index $\mathrm{RI}=1.12$. $\mathrm{CR}=\mathrm{CI} / \mathrm{RI}=0.0625<0.1$, the consistency is acceptable. Evaluation index weights are as Table 3:

\section{CASE STUDY}

This section studies an case of electric production. The production process can be divided into 3 stages. The production plan and the standard work time for each product at each stage are as Table 4.

The original layout is in the form of straight-line, where $\{N, S\}=\{\{4,4,4\},\{35,14,17\}\}$, and every line correspond to only one line of the next stage, e.g., if a product enters the 1 st line of the 1st stage, then it can only enters the 1 st line of the 2 nd and 3 rd stage.

After applying the model, we get a solution with a new $\{N, S\}=\{\{8,4,6\},\{17,14,12\}\}$, and the total number of workers is 264 , which is the same as before, but the average UPPH increase from 0.7878 to 1.4913 , which is a $89.3 \%$ improvement; the average cost decrease from 34.9 to 22.06 , as shown in Table $\mathbf{5}$.

\section{CONCLUSION}

This research builds a model on production line layout problem and offers simple yet effective solutions under various resource constraints. As for manufacturing companies with low margin and place much emphasis on cost, the model offers solutions to decrease the cost and improve the profit, and provides techniques to increase the companies' core competitiveness.

Table 2. Radom Index

\begin{tabular}{|c|c|c|c|c|c|c|c|c|c|}
\hline $\boldsymbol{n}$ & $\mathbf{1}$ & $\mathbf{2}$ & $\mathbf{3}$ & $\mathbf{4}$ & $\mathbf{5}$ & $\mathbf{6}$ & $\mathbf{7}$ & $\mathbf{8}$ & $\mathbf{9}$ \\
\hline \hline$R I$ & 0 & 0 & 0.58 & 0.90 & 1.12 & 1.24 & 1.32 & 1.41 & 1.45 \\
\hline
\end{tabular}

Table 3. Evaluation Index Weight

\begin{tabular}{|c|c|c|c|c|c|}
\hline Index & $\mathbf{I}_{\mathrm{PLI}}$ & $\mathbf{I}_{\mathrm{UAPH}}$ & $\mathbf{I}_{\mathrm{UPPH}}$ & $\mathbf{I}_{\mathrm{FR}}$ & $\mathbf{I}_{\mathrm{WIP}}$ \\
\hline \hline Weight & 0.1884 & 0.1874 & 0.0548 & 0.0332 & 0.5361 \\
\hline
\end{tabular}


Table 4. Production Plan and Standard Work Time

\begin{tabular}{|c|c|c|c|c|}
\hline No. & Quantity & \multicolumn{3}{|c|}{ Standard work time } \\
\hline 1 & 35 & 2016 & 896 & 1024 \\
\hline 2 & 444 & 554 & 299 & 345 \\
\hline 3 & 112 & 554 & 299 & 345 \\
\hline 5 & 450 & 554 & 299 & 276 \\
\hline 6 & 72 & 612 & 312 & 360 \\
\hline 7 & 54 & 648 & 360 & 360 \\
\hline 8 & 269 & 677 & 375 & 375 \\
\hline 12 & 2813 & 554 & 268 & 268 \\
\hline 13 & 187 & 524 & 268 & 268 \\
\hline 14 & 85 & 524 & 268 & 268 \\
\hline 15 & 63 & 524 & 268 & 247 \\
\hline 16 & 1204 & 588 & 299 & 276 \\
\hline 17 & 151 & 588 & 299 & 276 \\
\hline 18 & 48 & 588 & 299 & 276 \\
\hline
\end{tabular}

Table 5. Comparison of Improved and Original Layout

\begin{tabular}{|c|c|c|c|c|c|c|c|c|}
\hline & N1 & N2 & N3 & S1 & S2 & S3 & UPPH & Average Cost \\
\hline \hline Original & 4 & 4 & 4 & 35 & 14 & 17 & 0.7878 & 34.90 \\
\hline Improved & 8 & 4 & 6 & 17 & 14 & 12 & 1.4913 & 22.06 \\
\hline
\end{tabular}

\section{CONFLICT OF INTEREST}

The authors confirm that this article content has no conflicts of interest.

\section{ACKNOWLEDGEMENTS}

Declared none.

\section{REFERENCES}

[1] E.J. Muth, "Stochastic processes and their network representations associated with a production line queuing model", European Journal of Operational Research, vol. 15, no.1, pp. 63-83, 1984.

[2] J. Li and S.M, "Meerkov", Production systems engineering, 2008.

[3] G.C. Hadjinicola, "Manufacturing costs in serial production systems with rework", Journal of the Operational Research Society, vol. 61, no.2, pp. 342-351, 2009.

[4] H. Basali, B. Hussin, S.A. Asmai, N.K. Ibrahim, and A.S. Shibghatullah, "The integration of simple Markov model in solving single line", Information Technology in Asia, 2011.
[5] D.D. Spinellis and C.T. Papadopoulos, "A simulated annealing approach for buffer allocation inreliable production lines", Annals of Operations Research, vol. 93, no.1, pp. 373-384, 2000.

[6] S. Benjaafar, S.S. Heragu and S.A. Irani, "Next generation factory layouts: research challenges and recent progress", Interfaces, vol. 32 , no. 6 , pp. 58-76, 2002.

[7] M. Kuroda and T. Tomita, "Robust design of a cellular-line production system with unreliable facilities", Computers \& Industrial Engineering, vol. 48, no.3, pp.537-551, 2005.

[8] C.T. Papadopoulos, M.E.J. O'Kelly, M.J. Vidalis, and D. Spinellis, "Analysis and design of discrete part production lines", Springer, 2009.

[9] D. Quadt and H. Kuhn, "A taxonomy of flexible flow line scheduling procedures", European Journal of Operational Research, vol. 178, no. 3, pp. 686-698, 2007.

[10] J. Ribas, R. Leisten and J.M. Framinan, "Review and classification of hybrid flow shop scheduling problems from a production system and a solutions procedure perspective", Computers \& Operations Research, vol. 37, no.8, pp. 1439-1454, 2010.

[11] T. Urlings, R. Ruiz and T. Stutzle, "Shifting representation search for hybrid flexible flow line problems", European Journal of Operational Research, vol. 207, no. 2, pp. 1086-1095, 2010. 
[12] M. Haouari and L. Hidri, "On the hybrid flow shop scheduling problem", International Journal of Production Economics, vol. 113, no. 1, pp. 495-497, 2008.

[13] L. Hidri and M.Haouari, "Bounding strategies for the hybrid flow shop scheduling problem", Applied Mathematics and Computation, vol. 217 , no. 21 , pp. $8248-8263,2011$.
[14] D. Spinellis, C. Papadopoulos, and J.M. Smith, "Large production line optimization using simulated annealing", International Journal of Production Research, vol. 38, no. 3, pp. 509-541, 2000.

[15] S.M. Johnson, "Optimal two- and three-stage production schedules with setup times included", Naval Research Logistics Quarterly, vol. 1, no.1, pp. 61-68, 1954.

Received: August 13, 2013

(C) Zhao et al.; Licensee Bentham Open.

This is an open access article licensed under the terms of the Creative Commons Attribution Non-Commercial License (http://creativecommons.org/licenses/by-nc/3.0/) which permits unrestricted, non-commercial use, distribution and reproduction in any medium, provided the work is properly cited. 